\title{
Role of heat shock protein 70 in innate alloimmunity
}

\author{
Walter G. Land * \\ German Academy of Transplantation Medicine, Munich, Germany
}

\section{Edited by:}

Willem Van Eden, Utrecht University, Netherlands

Reviewed by:

Cristina Bonorino, Pontificia

Universidade Catolica do Rio Grande

do Sul, Brazil

Ruurd Van Der Zee, Universiteit

Utrecht, Netherlands

\section{*Correspondence:}

Walter G. Land, Köglweg 32, 82024

Taufkirchen, Germany.

e-mail:walterland@aol.com
This article briefly describes our own experience with the proven demonstration of heat shock protein 70 (HSP70) in reperfused renal allografts from brain-dead donors and reflects about its potential role as a typical damage-associated molecular pattern (DAMP) in the setting of innate alloimmunity. In fact, our group was able to demonstrate a dramatic upregulation of HSP70 expression after postischemic reperfusion of renal allografts. Of note, up-regulation of this stress protein expression, although to a lesser extent, was already observed after cold storage of the organ indicating that this molecule is already induced in the stressed organism of a brain-dead donor. However, whether or not the dramatic upregulation of HSP70 expression contributes to mounting an innate alloimmune response cannot be judged in view of these clinical findings. Nevertheless, HSP70, since generated in association with postischemic reperfusion-induced allograft injury, can be called a typical DAMP - as can every molecule be termed a DAMP that is generated in association with any stressful tissue injury regardless of its final positive or negative regulatory function within the innate immune response elicited by it. In fact, as we discuss in this article, the context-dependent, even contradistinctive activities of HSP70 reflect the biological phenomenon that, throughout evolution, mammals have developed an elaborate network of positive and negative regulatory mechanisms, which provide balance between defensive and protective measures against unwarranted destruction of the host. In this sense, up-regulated expression of HSP70 in an injured allograft might reflect a pure protective response against the severe oxidative injury of a reperfused donor organ. On the other hand, up-regulated expression of this stress protein in an injured allograft might reflect a (futile) attempt of the innate immune system to restore homeostasis with the aim to eliminate the "unwanted foreign allograft invader" by contributing to development of an adaptive alloimmune response. However, this adaptive immune response against donor histocompatibility alloantigens - in its evolutionary sense aimed to restore homeostasis is by no means protective from a recipient's view point but tragically ends up with allograft rejection. Indeed: in this sense, allograft rejection is the result of a fateful confusion by the immune system of danger and benefit!

Keywords: injury hypothesis, innate alloimmunity, DAMPs, HSP72

\section{INTRODUCTION: ALLOGRAFT INJURY-INDUCED INNATE ALLOIMMUNITY \\ FIRST CLUE TO THE EXISTENCE OF INNATE ALLOIMMUNITY: THE INJURY HYPOTHESIS (1994)}

Originally, the immune system was regarded as a host defense system that is primarily directed against invading pathogens with the aim to prevent/cure infection. Following the re-discovery of innate immunity in the $\mathrm{mid} /$ late $1990 \mathrm{~s}$, this concept was confirmed and extended: Infection of cells by microorganisms was seen to activate the innate immune system that elicits an inflammatory response merging into an adaptive immune response. The initial sensing of infection was recognized to be mediated by pattern recognition receptors (PRRs) in/on innate immune cells that play a pivotal role in the first line of this host defense system. PRRs were found to recognize distinct pathogen-derived motifs, the pathogen-associated molecular patterns (PAMPs) to initiate and regulate innate and adaptive immune responses (for review, see Kumar et al., 2011).
First notions that the immune system has evolved the capacity to detect any tissue injury in ways that stimulate the initiation and generation of adaptive immune responses to antigens were addressed and discussed by two hypotheses nearly simultaneously published early in 1994 (Land et al., 1994; Matzinger, 1994). These two hypotheses postulated that the adaptive immune system evolved to respond not only to pathogen-mediated "infectious" tissue injury per se but also to non-physiological cell death, tissue injury, or stress, as, for example, mediated by postischemic reperfusion injury (IRI). As a matter of fact, it was Matzinger (1994) who proposed her famous "Danger Hypothesis." Her model proposed on theoretical grounds - suggested that the primary driving force of the immune system is the need to detect and protect against danger. Danger, however, equals tissue destruction, that is, tissue injury. Our "Injury Hypothesis" - proposed on statistically significant data from a prospective clinical trial in kidney transplant patients (the "Munich SOD Trial") - discussed the possibility that it is the primary injury to an allograft 
that - via activation of antigen-presenting cells - induces pathways leading to an adaptive alloimmune response (Land et al., 1994). In fact, this hypothesis was based on our pivotal clinical observation that (antioxidative) treatment of a non-specific tissue injury [here: the reactive oxygen species (ROS)-mediated IRI to allografts] leads to a significant reduction in subsequent specific, adaptive immunity-mediated processes (here: reduction of alloimmune-mediated allograft rejection).

Under the line, the two hypotheses postulated the same scenario: The initial tissue injury, that is, the injurious inflammatory tissue environment, alerts the immune system, and is a mandatory prerequisite to mount an efficient adaptive immune response against foreign antigens.

\section{ROLE OF DAMAGE-ASSOCIATED MOLECULAR PATTERNS AND PATTERN RECOGNITION RECEPTORS IN OXIDATIVE INJURY - INDUCED ALLOGRAFT ("STERILE") INFLAMMATION}

Over the past decade, increasing evidence has been published in support of the notion that PRRs recognize non-infectious but injurious agents that can cause tissue damage (for reviews, see: Beutler, 2007; Manfredi et al., 2009; Chen and Nuñez, 2010; Bauernfeind et al., 2011; Jaeschke, 2011; Yanai et al., 2011). In this scenario, PRRs sense injury-induced, host-derived endogenous molecules in terms of damage-associated molecular patterns, that is, DAMPs, an acronym that was coined by us in analogy to PAMPs 8 years ago (Land, 2003a). These DAMPs are released following tissue injury or cell death and have similar functions as PAMPs in terms of their ability to activate pro-inflammatory pathways in innate immune cells.

Of note, ROS-mediated IRI to allografts can be regarded as a model of a non-pathogen-induced oxidative tissue injury that activates the innate immune system. In fact, an emerging role of innate immune events in adaptive alloimmunity-mediated allograft rejection ( $=$ innate alloimmunity) has been noted (for reviews, see Land, 2011a,b). However, the situation in the allograft setting is more complex as two individually different categories of DCs are involved: (1) donor-derived PRR-bearing innate immune cells such as DCs, vascular, and epithelial cells already residing in the transplant and (2) recipient-derived PRR-bearing innate immune cells such as DCs and neutrophils invading the allograft during reperfusion in the recipient. Moreover, various DAMPs such as high mobility group box 1 (HMGB1) and heat shock protein 70 (HSP70) are generated already in the brain-dead organ donor (Arbogast et al., 2002; Krüger et al., 2009; Kaminska et al., 2011), however, their up-regulated expression culminate during allograft reperfusion in the recipient (Arbogast et al., 2002; Kaczorowski et al., 2009; Klune and Tsung, 2010). Indeed, according to new insights into mechanisms of innate immunity, a brain-dead organism, characterized by the demonstration of DAMPs, PRRs, and circulating cytokines, may be defined as an acute systemic innate immunity-mediated autoinflammatory syndrome (Land, 2011b). To add yet another level of complexity in the allograft setting, it can be assumed that further DAMPs are released from necrotic cells caused by allograft reperfusion injury which may include nucleic acids, oxidation-specific DAMPs such as thioredoxin (TRX)-interacting protein (TXNIP) and purine metabolites such as ATP (Schröder et al., 2010; Bours et al., 2011; Kis-Toth et al., 2011). In addition to those DAMPs derived from an intracellular source, IRI leads to release of extracellular located DAMPs such as extracellular matrix fragments including hyaluronan and heparan sulfate. The prototypical PRRs recognizing all those various DAMPs include Toll-like receptors (TLRs) such as TLR2 and TLR4 and the receptor for advanced glycation end-products (RAGE) as well as PRRs able to recognize nucleic acids such as RIG-I-like receptors (RLRs) and AIM2-like receptors (for review, see Land, 2011e).

There is a special role for the cytosolic nucleotide-binding domain leucine-rich repeats (NLR) receptors because one of its members represents the core structure of the NOD, LRR, and pyrin domain-containing 3 (NLRP3) inflammasome, the most widely studied inflammasome now known to be activated by various DAMPs (for review, see Bauernfeind et al., 2011). In fact, the wellknown creation of "sterile" inflammation in reperfused organs (and solid allografts have to be included here) is now believed to be induced by inflammasomes. Most important in regard to the topic of IRI are recent studies in both Nlrp3- and ASC-deficient mice clearly demonstrating that the NLRP3 inflammasome contributes to the IRI-mediated acute inflammatory response (Iyer et al., 2009). These studies revealed that non-lethal renal IRI results in a significant up-regulation of Nlrp3 gene expression, which is accompanied by pronounced acute tubular necrosis that is similar between wild-type (WT) and Nlrp3-deficient animals. Importantly, the studies showed that Nlrp3-deficiency protected animals from lethal renal IRI. Similarly, $A S C$-deficiency protected animals from lethal renal IRI, although the difference, compared to WT mice, was less pronounced than in Nlrp3-deficient mice.

Activation of the NLRP3 inflammasome is currently thought to consist of two steps: a first priming step that consists of stimulation of PRRs which leads to the up-regulation of NLRP3 expression and also induces pro-IL-1 $\beta$ expression. Stimuli priming NLRP3 appear to include all ligands for TLRs, RLRs, and NLRs, that lead to enhanced NLRP3 expression (Bauernfeind et al., 2011). Thus, theoretically, HSP70, as a ligand of TLR4 and TLR2, can be counted to those priming DAMPs (The LPS-contamination debate in regard to TLR2 and TLR4 is not dealt with here but discussed elsewhere; Land, 2011e). The second step consists of the activation of NLRP3 itself which is distinct from this initial priming step. Three presumably distinct pathways have been postulated that can lead to the activation of NLRP3 (activation step): (1) extracellular ATP that is often seen to be largely increased during cell death, can bind to purinergic receptor P2X7 and leads to NLRP3 activation via ion flux effects (including intracellular $\mathrm{K}^{+}$depletion) mediated by the P2X7-receptor-associated hemi-channel pannexin-1; (2) endocytosis of sterile particulates, such as cholesterol crystals, resulting in lysosomal disintegration which leads to the leakage of lysosomal enzymes into the cytosol (activation of the protease cathepsin B); and (3) generation of ROS during cellular stress or death leading to the activation of NLRP3 through the release of TXNIP from thioredoxin and then binds to NLRP3 (for review, see Bauernfeind et al., 2011). Although the role of ROS in NLRP3 activation is still not quite clear, this potential activation mechanism is of considerable attraction in regard to ROS-mediated reperfusion injury to allografts. 


\section{ALLOGRAFT INJURY-INDUCED, DAMPS-MEDIATED GENERATION OF DONOR-AND RECIPIENT-DERIVED IMMUNOSTIMULATORY DCS}

In fact, growing evidence suggests that allograft injury induces innate immune events which precede adaptive alloimmunity. Within an innate immune-mediated intragraft inflammatory milieu, donor- and recipient-derived DCs get activated, migrate to the secondary lymphoid tissue of the recipient to translate innate immunity to an adaptive alloimmune response. The generation of these immunostimulatory DCs is the result of innate immune pathways initiated and induced by various DAMPs which, as reviewed (Land, 2011b), may be divided into four different classes of DAMPs: (1) class I DAMPs such as HMGB1 and HSP70 that, when recognized by PRRs of immature DCs (iDCs), trigger their activation to immunostimulatory DCs; (2) class II DAMPs such as MHC class I chain-related molecules A and B that, when recognized by special activating receptors (NKG2D) on innate lymphocytes may contribute to generation of immunostimulatory DCs in terms of a cross-talk; (3) class III DAMPs such as TXNIP and extracellular ATP that are recognized by PRRs involved in the activation of the NLRP3 inflammasome, and (4) class IV DAMPs in terms of neoantigens that are recognized by pre-existing natural immunoglobulin $\mathrm{M}$ antibodies, which - via complement activation - are able to aggravate the oxidative tissue injury and, thereby, may indirectly promote metamorphosis of iDCs into immunostimulatory DCs.

In fact, there seems to be a complex collaboration between various membrane-bound and cytosolic PRRs on one side and their different cognate DAMPs on the other side which results in those well-known vigorous innate alloimmune responses. Most likely, this complex orchestration of intragraft DAMP-PRR interactions may finely regulate the maturation process of donor- and recipient-derived iDCs into immunostimulatory DCs, that, via processes of direct and indirect allorecognition, may stimulate naïve $\mathrm{T}$ cells of the recipient, thereby mounting, and fine-tuning adaptive alloimmune responses of various intensities.

\section{ROLE OF HEAT SHOCK PROTEIN 70 IN INNATE ALLOIMMUNITY \\ DONOR BRAIN DEATH-MEDIATED AND REPERFUSION INJURY-INDUCED UP-REGULATION OF HSP70 EXPRESSION IN HUMAN ALLOGRAFTS}

The first DAMP shown to be involved in the setting of clinical organ transplantation was the inducible HSP70 that was upregulated following renal allograft reperfusion injury, that is, a condition in which contamination with pathogen-derived exogenous ligands of PRRs can be totally ruled out (Arbogast et al., 2002). In fact, our group was able to demonstrate a dramatic up-regulation of HSP70 expression after postischemic reperfusion of renal allografts from deceased (brain-dead) donors (Figure 1). Interestingly, up-regulation of HSP70 expression, although to a lesser extent, was already observed after cold storage of the organ indicating that this stress protein is already induced in the stressed organism of a brain-dead donor (Figure 1). These findings prompted us to discuss a role of HSP70 in innate alloimmunity by proposing that HSPs (1) in their function as endogenous ligands of TLRs may lead to DC maturation and (2) in their function as chaperokines may facilitate cross-presentation of HSP-chaperoned allopeptides

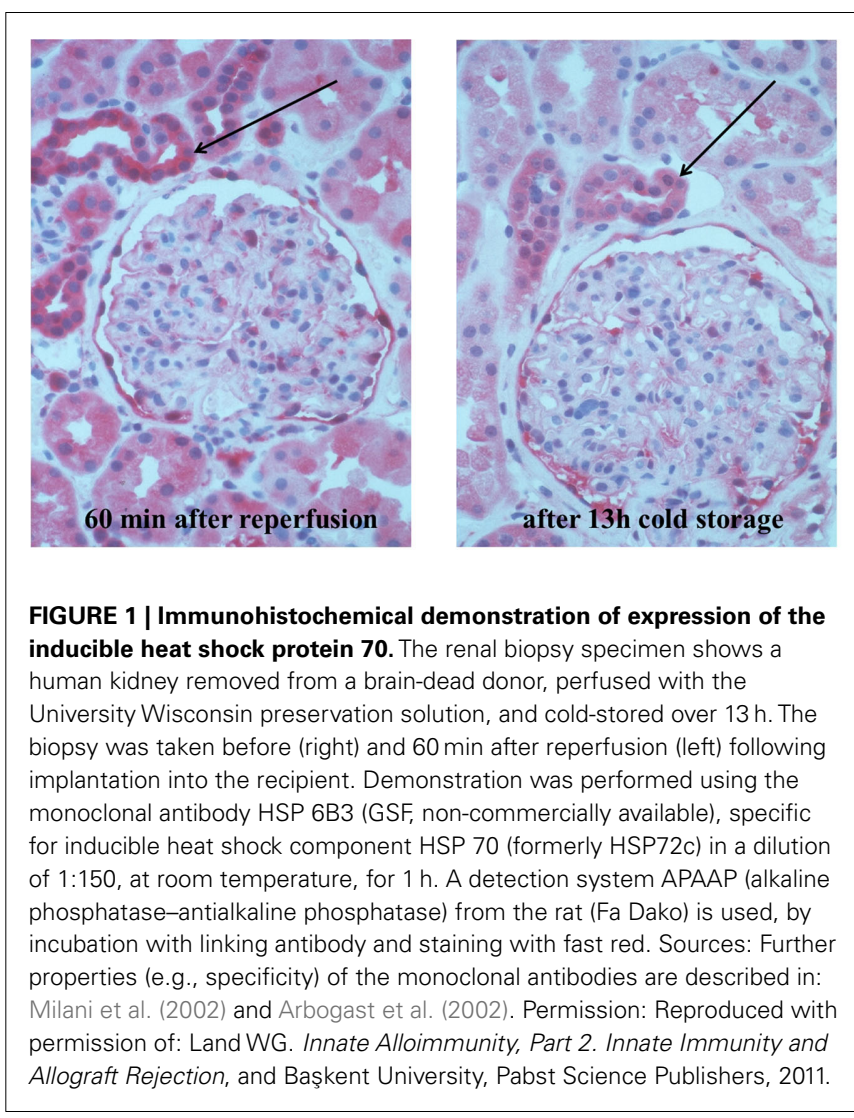

on MHC class I molecules of recipient-derived DCs (Land, 2002, 2003b). Whether or not dramatic up-regulation of HSP70 expression in an allograft contributes to mounting an innate alloimmune response cannot be judged in view of these clinical findings alone, but a brief look at the more recent literature may help in interpreting our earlier findings.

\section{ROLE OF HSP70 FAMILY MEMBERS IN ENHANCING ADAPTIVE IMMUNE RESPONSES}

In general, extracellular HSPs released from damaged cells are immunogenic and have been shown to enhance TH1 adaptive immune responses and, therefore, have been used for improved vaccination procedures against infectious diseases and cancer (for review, see Binder, 2006). For example, a novel HSP70 family member, termed Hsp70-like protein 1 (Hsp70L1) was recently identified as a potent TH1 polarizing adjuvant that contributes to antitumor immune responses (Fang et al., 2011). In another line of experiments on a fusion cell model (DC and tumor cell fusion), efficient cytotoxic T lymphocyte productivity of modified fusion cells was demonstrated and supposed to be due to an up-regulation of HSP70 (Koide et al., 2009).

Such enhancement of adaptive immune responses by HSP70 is thought to be preferentially mediated by its capacity to promote maturation of immunostimulatory DCs as well as to enhance cross-presentation of peptide antigens, that is, a crucial pathway of exogenous antigen presentation on the HLA class I molecules by DCs, enabling adaptive antiviral or antitumor responses. 
In fact, in earlier studies, it was already shown that human HSP70 preparations are able to induce DC maturation (Kuppner et al., 2001; Bethke et al., 2002). In a more recent study on an in vitro-induced ischemia model, bone marrow-derived DCs were shown to augment allogeneic T-cell proliferation as well as the interferon-gamma response (Jurewicz et al., 2010). In this study, TLR4 ligation was also shown to occur in ischemic DCs, most likely - as concluded by the authors - in response to HSP70, which was found to be elevated in DCs after ischemic injury.

In addition to contributing to the generation of immunostimulatory DCs in terms of a ligand of TLR2/4, extracellular HSP70 has been shown in models of antitumor T-cell responses to promote the cross-presentation of peptide antigens to MHC class I molecules in DCs, leading to efficient induction of antigenspecific cytotoxic T-lymphocytes (Castelli et al., 2001; Srivastava, 2002; Ueda et al., 2004). Of particular importance for the discussion of a role of cross-presentation in innate alloimmunity are recent studies demonstrating that HSP70 binds HLA class I and class II epitope precursors, which further strengthens the role of HSP70 in the HLA class I and class II antigen presentation process (Stocki et al., 2010). In fact, these studies lent support to a more general characteristic of the HSP70-chaperoned peptides and suggest involvement of HSP70 as a more general phenomenon in the antigen presentation process.

\section{HEAT SHOCK PROTEINS AND ALLOGRAFT SURVIVAL}

Most interesting for our early assumption in 2002/2003 that HSP70 may be involved in innate alloimmunity would be the provision of data from experimental studies on allograft models. However, there are only a few such data published so far and, unfortunately, they are even controversial. Thus, it could be shown that targeted gene disruption of the $h s p 72$ gene in donor tissue is associated with significantly but modestly prolonged rejectionfree survival in a murine skin allograft model, the difference between $h s p 72$-deficient mice and controls being 3 days only (Oh et al., 2004). Nonetheless, these experimental data suggest that HSP70 in terms of a pro-inflammatory acting DAMP contributes to an innate alloimmune response although only in a moderate way.

On the other hand, data from another experimental allograft model indicate that HSP70 in terms of an anti-inflammatory acting DAMP may even exert a beneficial effect on allograft survival, although again quite modestly (Borges et al., 2010). In this study, Mycobacterium tuberculosis HSP70 (TBHSP70) was shown to inhibit allograft rejection in a skin allograft model in which the donor skin was immersed in a PBS solution containing TBHSP70 before transplantation. Interestingly, however, TBHSP70-induced prolongation of skin allograft survival was associated with the demonstration of $\mathrm{T}$ regulatory cells (Tregs), a finding supposed by the authors to mediate the graftprolonging effect. Interestingly, the authors discuss (although not conclusive from their observations) whether or not the Tregs observed in their studies are specific for TBHSP70, by referring to growing evidence in support of the notion that HSPs possess inherent immunoregulatory properties (van Eden et al., 2005).

\section{SYNOPSIS}

In my opinion, HSPs, since generated in association with any stressful tissue injury, can be called typical damage-associated molecular patterns, that is, DAMPs. Their evolutionarily determined, inherent protective role in concert with a large panoply of other molecules in commission of the innate immune system lies in defense against any cell/tissue injury (including elimination of the injurious agent concerned, e.g., viruses, tumor cells).

In regard to innate alloimmunity, it can be concluded from our own experience that the expression of HSP70 is moderately increased in kidneys from brain-dead donors but dramatically upregulated after IRI to human renal allografts, thus, fulfilling the criteria of a DAMP. No more, no less. However, the exact biological activity of HSP70 in innate alloimmunity remains elusive. Without addressing the "contamination debate" here, one may discuss that, in the excessively inflammatory milieu of a postischemically reperfused allograft, this DAMP - in concert and collaboration with a variety of other DAMPs such as HMGB1 and nucleic acids released from necrotic graft cells - may operate as an immunity-initiating, -promoting, and -facilitating stress protein to allograft rejection [the originally protective role of HSPs here is being converted to a harmful response from a recipient's point of view, caused by a fateful confusion of "invading" foreign antigens (alloantigens) within an injured organ and foreign antigens derived from invading dangerous pathogens]. Potential pro-inflammatory properties of HSP70 may lie in its capacities (1) to act as a "priming" DAMP of the NLRP3 inflammasome contributing to the typical inflammatory environment of a reperfused allograft, (2) to contribute to maturation of donor- and recipient-derived DCs by engagement with TLR4/2, and (3) to intensify re-presentation of allopeptides within donor MHC class II molecules on donor- and recipientderived DCs as well as promote cross-presentation of allopeptides within donor MHC class I molecules on recipient-derived DCs.

In contrast, in a non-injurious or weakly injurious tissue microenvironment, for example, when HSP70 is added to skin grafts immersed in culture prior to transplantation (Borges et al., 2010), this DAMP may elicit negative regulation mechanisms in graft-containing antigen-presenting cells on the level of TLR signaling. As known, in order to prevent catastrophic host immune overreactions, such negative feedback regulations exist that control the intensity and duration of TLR-triggered innate immune responses - best known as the classic negative regulation in response to LPS, the "LPS or endotoxin tolerance." In fact, molecular feedback inhibitors operating at different levels of TLRsignaling pathways can be divided into three major groups: extracellular regulators such as soluble decoy TLRs, transmembrane protein regulators such as the suppressor of tumorigenicity 2, and intracellular negative regulators such as IL-1 receptor-associated kinase-M (IRAK-M) and members of the suppressors of cytokine signaling (SOCS) family (for review, see Land, 2011d).

For example, the well-known anti-inflammatory effect of preconditioning procedures including heat preconditioning associated with the expression of HSP70 (Jo et al., 2006) has been thought to be due to elicitation of regulatory feedback inhibitors of TLR signaling (for review, see also Karikó et al., 2004). In fact, in earlier studies, it was demonstrated that prior exposure to HSP70, here the Toxoplasma gondii-derived HSP70, 
induces a hyporesponse in macrophages to subsequent stimulation with this HSP70 by expression of SOCS1 via TLR4 (Mun et al., 2005). Of note, as shown in more recent studies on a model of hepatic IRI, preconditioning using the TLR4 agonist LPS elicited the up-regulation of specific negative regulators including SOCS1 and IRAK-M in the TLR4-signaling pathway (Sano et al., 2011). Impressively, molecules operating in those regulatory feedback loops have been shown to also prevent DC maturation, that is, to promote generation of tolerogenic DCs known to induce Tregs (for reviews, see Maldonado and von Andrian, 2010; Land, 2011c). Thus, data from an experimental model of SOCS1-gene overexpression in bone marrowderived DCs showed that SOCS1 inhibits DC maturation and induces generation of regulatory DCs, which - via generation of $\mathrm{CD} 4^{+} \mathrm{CD} 25^{+}$Tregs - resulted in prolongation of allograft survival (Fu et al., 2009). First evidence of a similar feedback inhibiting effect of SOCS2 on TLR-induced activation in human

\section{REFERENCES}

Arbogast, H., Arbogast, S., Fertmann, J., and Land, W. (2002). Expression of heat shock proteins in cadaveric human renal allografts - a role in activation of innate immunity? Transplantation 74(Suppl.), 266.

Bauernfeind, F., Ablasser, A., Bartok, E., Kim, S., Schmid-Burgk, J., Cavlar, T., and Hornung, V. (2011). Inflammasomes: current understanding and open questions. Cell. Mol. Life Sci. 68, 765-783.

Bethke, K., Staib, F., Distler, M., Schmitt, U., Jonuleit, H., Enk, A. H., Galle, P. R., and Heike, M. (2002). Different efficiency of heat shock proteins (HSP) to activate human monocytes and dendritic cells: superiority of HSP60. J. Immunol. 169, 6141-6148.

Beutler, B. (2007). Neo-ligands for innate immune receptors and the etiology of sterile inflammatory disease. Immunol. Rev. 220, 113-128.

Binder, R. J. (2006). Heat shock protein vaccines: from bench to bedside. Int Rev. Immunol. 25, 353-375.

Borges, T. J., Porto, B. N., Teixeira, C. A., Rodrigues, M., Machado, F. D., Ornaghi, A. P., de Souza, A. P., Maito, F., Pavanelli, W. R., Silva, J. S., and Bonorino, C. (2010). Prolonged survival of allografts induced by mycobacterial HSP70 is dependent on $\mathrm{CD} 4 \mathrm{CD} 25$ regulatory T cells. PLoS ONE 5, e14264. doi:10.1371/journal.pone.0014264

Bours, M. J., Dagnelie, P. C., Giuliani, A. L., Wesselius, A., and Di Virgilio, F. (2011). P2 receptors and extracellular ATP: a novel homeostatic pathway in inflammation. Front. Biosci. (Schol. Ed). 3, 1443-1456.

Castelli, C., Ciupitu, A. M., Rini, F., Rivoltini, L., Mazzocchi, A., Kiessling, R., and Parmiani, G. (2001). Human heat shock protein
70 peptide complexes specifically activate antimelanoma T cells. Cancer Res. 61, 222-227.

Chen, G. Y., and Nuñez, G. (2010). Sterile inflammation: sensing and reacting to damage. Nat. Rev. Immunol. $10,826-837$.

Fang, H., Wu, Y., Huang, X., Wang, W., Ang, B., Cao, X., and Wan, T. (2011). Toll-like receptor 4 (TLR4) is essential for Hsp70-like protein 1 (HSP70L1) to activate dendritic cells and induce Thl response. J. Biol. Chem. 286, 30393-30400.

Fu, H., Song, S., Liu, F., Ni, Z., Tang, Y., Shen, X., Xiao, L., Ding, G., and Wang, Q. (2009). Dendritic cells transduced with SOCS1 gene exhibit regulatory DC properties and prolong allograft survival. Cell. Mol. Immunol. 6, 87-95.

Iyer, S., Pulskensc, W., Sadler, J., Butter, L. M., Teske, G. J., Ulland, T. K., Eisenbarth, S. C., Florquin, S., Flavell, R. A., Leemans, J. C., and Sutterwala, F. S. (2009). Necrotic cells trigger a sterile inflammatory response through the Nlrp3 inflammasome. Proc. Nat. Acad. Sci. U.S.A. 106, 20388-20393.

Jaeschke, H. (2011). Reactive oxygen and mechanisms of inflammatory liver injury: present concepts. J. Gastroenterol. Hepatol. 26(Suppl. 1), 173-179.

Jo, S. K., Ko, G. J., Boo, C. S., Cho, W. Y., and Kim, H. K. (2006). Heat preconditioning attenuates renal injury in ischemic ARF in rats: role of heatshock protein 70 on NF-kappaBmediated inflammation and on tubular cell injury. J. Am. Soc. Nephrol. 17, 3082-3092.

Jurewicz, M., Takakura, A., Augello, A., Naini, S. M., Ichimura, T., ZandiNejad, K., and Abdi, R. (2010). Ischemic injury enhances dendritic

monocyte-derived DCs has also been recently reported (Posselt et al., 2011).

Altogether, the context-dependent, even contradistinctive activities of HSP70 reflect the biological phenomenon that, throughout evolution, mammals have developed an elaborate network of positive and negative regulatory mechanisms, which provide balance between defensive measures against dangerous bacterial and viral pathogens and protective measures against unwarranted destruction of the host by the activated immune system. Fine-tuning of TLR signaling in amplitude, space, time, and character is a key aspect of inflammatory reactions in health, homeostasis, and pathology. What is becoming more and more apparent is that positive and negative regulators within immune responses do not work as a single entity, but rather, similar to an orchestral score, each component is reliant on its other tools such as HSP70 to produce a harmonious melody instead of a crashing cacophony.

cell immunogenicity via TLR4 and NF-kappa B activation. J. Immunol. 184, 2939-2948.

Kaczorowski, D. J., Nakao, A., Vallabhaneni, R., Mollen, K. P., Sugimoto, R., Kohmoto, J., Zuckerbraun, B. S., McCurry, K. R., and Billiar, T. R. (2009). Mechanisms of Toll-like receptor 4 (TLR4) mediated inflammation after cold ischemia/reperfusion in the heart. Transplantation 87, 1455-1463.

Kaminska, D., Koscielska-Kasprzak, K. Drulis-Fajdasz, D., Halon, A., Polak, W., Chudoba, P., Janczak, D., Mazanowska, O., Patrzalek, D., and Klinger, M. (2011). Kidney ischemic injury genes expressed after donor brain death are predictive for the outcome of kidney transplantation. Transplant. Proc. 43, 2891-2894.

Karikó, K., Weissman, D., and Welsh, F. A. (2004). Inhibition of Toll-like receptor and cytokine signaling - a unifying theme in ischemic tolerance. J. Cereb. Blood Flow Metab. 24, 1288-1304.

Kis-Toth, K., Szanto, A., Thai, T. H., and Tsokos, G. C. (2011). Cytosolic DNA-activated human dendritic cells are potent activators of the adaptive immune response. J. Immunol. 187, 1222-1234.

Klune, J. R., and Tsung, A. (2010). Molecular biology of liver ischemia/reperfusion injury: established mechanisms and recent advancements. Surg. Clin. North Am. 90, 665-677.

Koide, T., Iinuma, H., and Fukushima, R. (2009). Efficient CTL productivity of modified fusion cells by increase of heat shock protein 70 . Oncol. Rep. 21, 737-746.

Krüger, B., Krick, S., Dhillon, N., Lerner, S. M., Ames, S., Bromberg, J. S., Lin, M., Walsh, L., Vella, J., Fischereder,
M., Krämer, B. K., Colvin, R. B., Heeger, P. S., Murphy, B. T., and Schröppel, B. (2009). Donor Toll-like receptor 4 contributes to ischemia and reperfusion injury following human kidney transplantation. Proc. Natl. Acad. Sci. U.S.A. 106, 3390-3395.

Kumar, H., Kawai, T., and Akira, S. (2011). Pathogen recognition by the innate immune system. Int. Rev. Immunol. 30, 16-34.

Kuppner, M. C., Gastpar, R., Gelwer, S., Nössner, E., Ochmann, O., Scharner, A., and Issels, R. D. (2001). The role of heat shock protein (hsp70) in dendritic cell maturation: hsp70 induces the maturation of immature dendritic cells but reduces DC differentiation from monocyte precursors. Eur. J. Immunol. 31, 1602-1609.

Land, W. (2002). Postischemic reperfusion injury to allografts - a case for "innate immunity"? Eur. Surg. Res. 34, 160-169.

Land, W. (2003a). Allograft injury mediated by reactive oxygen species: from conserved proteins of Drosophila to acute and chronic rejection of human transplants. Part III: interaction of (oxidative) stress-induced heat shock proteins with Toll-like receptor-bearing cells of innate immunity and its consequences for the development of acute and chronic allograft rejection. Transplant. Rev. 17, 67-86.

Land, W. (2003b). Allograft injury mediated by reactive oxygen species: from conserved proteins of Drosophila to acute and chronic rejection of human transplants. Part II: role of reactive oxygen species in the induction of the heat shock response as a regulator of innate immunity. Transplant. Rev. 17, 31-44. 
Land, W., Schneeberger, H., Schleibner, S., Illner, W. D., Abendroth, D., Rutili, G., Arfors, K. E., and Messmer, K. (1994). The beneficial effect of human recombinant superoxide dismutase on acute and chronic rejection events in recipients of cadaveric renal transplants. Transplantation 57, 211-217.

Land, W. G. (2011a). Emerging role of innate immunity in organ transplantation. Part I: evolution of innate immunity and oxidative allograft injury. Transplant. Rev. (Orlando). doi: $\quad 10.1016 / j . t r r e .2011 .05 .001$. [Epub ahead of print].

Land, W. G. (2011b). Emerging role of innate immunity in organ transplantation. Part II: Potential of DAMPs to generate immunostimulatory dendritic cells. Transplant. Rev. (Orlando). doi: 10.1016/jtrre.2011.02.003. [Epub ahead of print].

Land, W. G. (2011c). Emerging role of innate immunity in organ transplantation. Part III: the quest for transplant tolerance via prevention of oxidative allograft injury and its consequences. Transplant. Rev. (Orlando). doi: 10.1016/j.trre.2011.07.001. [Epub ahead of print].

Land, W. G. (2011d). "Negative regulation of TLR-signaling pathways," in Innate Alloimmunity, Part I: Innate Immunity and Host Defence, ed. W. G. Land (Lengerich: Başkent University, Pabst Science Publishers), 267-285.
Land, W. G. (2011e). "Oxidative injury-induced, damage-associated molecular pattern molecules and their pattern recognition receptors," in Innate Alloimmunity, Part II: Innate Immunity and Allograft Rejection, ed. W. G. Land (Lengerich: Başkent University, Pabst Science Publishers), 229-337.

Maldonado, R. A., and von Andrian, U. H. (2010). How tolerogenic dendritic cells induce regulatory $\mathrm{T}$ cells. Adv. Immunol. 108, 111-165.

Manfredi, A. A., Capobianco, A., Bianchi, M. E., and Rovere-Querini, P. (2009). Regulation of dendriticand T-cell fate by injury-associated endogenous signals. Crit. Rev. Immunol. 29, 69-86.

Matzinger, P. (1994). Tolerance, danger, and the extended family. Annu. Rev. Immunol. 12, 991-1045.

Milani, V., Noessner, E., Ghose, S., Kuppner, M., Ahrens, B., Scharner, A., Gastpar, R., and Issels, R. D. (2002). Heat shock protein 70 role in antigen presentation and immune stimulation. Int. J. Hyperthermia 18, 563.

Mun, H. S., Aosai, F., Norose, K., Piao, L. X., Fang, H., Akira, S., and Yano, A. (2005). Toll-like receptor 4 mediates tolerance in macrophages stimulated with Toxoplasma gondii-derived heat shock protein 70. Infect. Immun. 73, 4634-4642.

Oh, K. H., Kim, J. Y., Kim, D., Lee, E. M., Oh, H. Y., Seo, J. S., Han, J. S., Kim, S., Lee, J. S., and Ahn, C. (2004). Targeted gene disruption of the heat shock protein 72 gene (hsp70.1) in the donor tissue is associated with a prolonged rejection-free survival in the murine skin allograft model. Transpl. Immunol. 13, 273-281.

Posselt, G., Schwarz, H., Duschl, A., and Horejs-Hoeck, J. (2011). Suppressor of cytokine signaling 2 is a feedback inhibitor of TLR-induced activation in human monocyte-derived dendritic cells. J. Immunol. 187, 2875-2884.

Sano, T., Izuishi, K., Hossain, M. A. Inoue, T., Kakinoki, K., Hagiike, M. Okano, K., Masaki, T., and Suzuki, Y. (2011). Hepatic preconditioning using lipopolysaccharide: association with specific negative regulators of the Toll-like receptor 4 signaling pathway. Transplantation 91, 1082-1089.

Schröder, K., Zhou, R., and Tschopp J. (2010). The NLRP3 inflammasome: a sensor for metabolic danger? Science 327, 296-300.

Srivastava, P. (2002). Interaction of heat shock proteins with peptides and antigen presenting cells: chaperoning of the innate and adaptive immune responses. Annu. Rev. Immunol. 20, 395-425.

Stocki, P., Morris, N. J., Preisinger, C. Wang, X. N., Kolch, W., Multhoff, G. and Dickinson, A. M. (2010). Identification of potential HLA class I and class II epitope precursors associated with heat shock protein 70 (HSPA). Cell Stress Chaperones 15, 729-741.

Ueda, G., Tamura, Y., Hirai, I., Kamiguchi, K., Ichimiya, S., Torigoe, T., Hiratsuka, H., Sunakawa, H. and Sato, N. (2004). Tumor-derived heat shock protein 70-pulsed dendritic cells elicit tumor-specific cytotoxic $\mathrm{T}$ lymphocytes (CTLs) and tumor immunity. Cancer Sci. 95, 248-253.

van Eden, W., van der Zee, R., and Prakken, B. (2005). Heat-shock proteins induce $\mathrm{T}$ cell regulation of chronic inflammation. Nat. Rev. Immunol. 5, 318-330.

Yanai, H., Ban, T., and Taniguchi, T. (2011). Essential role of highmobility group box proteins in nucleic acid-mediated innate immune responses. J. Intern. Med. 270, 301-308.

Conflict of Interest Statement: The author declares that the research was conducted in the absence of any commercial or financial relationships that could be construed as a potential conflict of interest.

Received: 24 October 2011; accepted: 19 December 2011; published online: 06 January 2012.

Citation: Land WG (2012) Role of heat shock protein 70 in innate alloimmunity. Front. Immun. 2:89. doi: 10.3389/fimmu.2011.00089

This article was submitted to Frontiers in Inflammation, a specialty of Frontiers in Immunology.

Copyright (c) 2012 Land. This is an open-access article distributed under the terms of the Creative Commons Attribution Non Commercial License, which permits non-commercial use, distribution, and reproduction in other forums, provided the original authors and source are credited. 